\title{
The MarR family regulator OsbR controls oxidative stress response, anaerobic nitrate respiration, and biofilm formation in Chromobacterium violaceum
}

\author{
Júlia A. Alves ${ }^{1}$, Maristela Previato-Mello ${ }^{1}$, Kelly C. M. Barroso ${ }^{1}$, Tie Koide ${ }^{2}$ and José F. da Silva Neto ${ }^{1 *}$
}

\begin{abstract}
Background: Chromobacterium violaceum is an environmental opportunistic pathogen that causes rare but deadly infections in humans. The transcriptional regulators that $C$. violaceum uses to sense and respond to environmental cues remain largely unknown.

Results: Here, we described a novel transcriptional regulator in C. violaceum belonging to the MarR family that we named OsbR (oxidative stress response and biofilm formation regulator). Transcriptome profiling by DNA microarray using strains with deletion or overexpression of osbR showed that OsbR exerts a global regulatory role in C. violaceum, regulating genes involved in oxidative stress response, nitrate reduction, biofilm formation, and several metabolic pathways. EMSA assays showed that OsbR binds to the promoter regions of several OsbR-regulated genes, and the in vitro DNA binding activity was inhibited by oxidants. We demonstrated that the overexpression of os $R$ R caused activation of ohrA even in the presence of the repressor OhrR, which resulted in improved growth under organic hydroperoxide treatment, as seem by growth curve assays. We showed that the proper regulation of the nar genes by OsbR ensures optimal growth of C. violaceum under anaerobic conditions by tuning the reduction of nitrate to nitrite. Finally, the osbR overexpressing strain showed a reduction in biofilm formation, and this phenotype correlated with the OsbR-mediated repression of two gene clusters encoding putative adhesins.
\end{abstract}

Conclusions: Together, our data indicated that OsbR is a MarR-type regulator that controls the expression of a large number of genes in C. violaceum, thereby contributing to oxidative stress defense (ohrA/ohrR), anaerobic respiration (nark1K2 and narGHJI), and biofilm formation (putative RTX adhesins).

Keywords: Chromobacterium violaceum, MarR family regulator, OsbR regulon, Transcriptome analysis, Oxidative stress, Biofilm formation, Nitrate respiration

*Correspondence: jfsneto@usp.br

${ }^{1}$ Departamento de Biologia Celular e Molecular e Bioagentes

Patogênicos, Faculdade de Medicina de Ribeirão Preto, Universidade de

São Paulo, Ribeirão Preto, SP, Brazil

Full list of author information is available at the end of the article

\begin{abstract}
Background
Chromobacterium violaceum is a Gram-negative betaproteobacterium found in tropical and subtropical ecosystems around the world, primarily in water and soil samples $[1,2]$. C. violaceum strains produce several enzymes and secondary metabolites with biotechnological interest, including quitinases, hydrogen cyanide, siderophores, the antitumoral depsipeptide FR901228, and antibiotics, such as violacein. The purple pigment
\end{abstract}


violacein has in vitro activity against eukaryotic cells and Gram-positive bacteria [1,3]. From the environmental reservoir, $C$. violaceum can cause rare but deadly opportunistic infections in humans and other animals, entering the hosts mainly through skin lesions. The resulting disease manifests as fatal septicemia and abscesses in the lung and liver [2, 4]. Similar to other environmental pathogens, C. violaceum has complex regulatory systems and versatile metabolic capacities to survive under several stress conditions [5-7]. For example, genome sequence analyses indicate that $C$. violaceum possesses a nitrate reductase (Nar) to reduce nitrate to nitrite to obtain energy under anaerobic conditions $[5,6]$. However, the transcriptional regulators involved in C. violaceum adaptation to diverse environments remain poorly investigated.

The MarR (multiple antibiotic resistance regulator) family of transcriptional regulators, widespread in bacteria and archaea, includes proteins involved in several bacterial processes, such as antibiotic resistance, virulence control, oxidative stress response, and catabolism of aromatic compounds [8-13]. Many MarR-type regulators act as transcriptional repressors that dissociate from DNA upon binding of small ligands or oxidation of conserved Cys residues [10-12]. One prototypical redox-sensing MarR regulator is OhrR, a Cys-based redox sensor that, upon oxidation by organic hydroperoxides, releases the transcription of $o h r A$, encoding a Cys-based peroxidase [14-17]. Other redox-sensing MarR regulators control large regulons and multiple bacterial responses in addition to antioxidant defense. For instance, OspR of Pseudomonas aeruginosa, an OhrR homolog, regulates pigment production, antibiotic resistance, virulence, and antioxidant enzymes [18, 19]; MgrA of Staphylococcus aureus is a global regulator of virulence, antibiotic resistance, biofilm, and clumping [20,21]. Several homologs of OspR and MgrA have been characterized, including SarZ of S. aureus, MosR of Mycobacterium tuberculosis, AbfR of Staphylococcus epidermidis, BmoR of Bacteroides fragilis, and AsrR of Enterococcus faecium [22-26]. The homolog pairs OhrR/OspR in P. aeruginosa and MgrA/ SarZ in S. aureus control distinct and overlapping functions $[18,22]$.

Based on the genome sequence, $C$. violaceum has more than two hundred predicted transcriptional regulators grouped in several families, including the MarR family with at least fifteen members [6]. However, only a few transcriptional regulators have been characterized in C. violaceum. The master regulator CilA activates most genes from the Chromobacterium pathogenicity islands 1 and $1 \mathrm{a}(\mathrm{Cpi}-1 /-1 \mathrm{a})$ that encode a type III secretion system required for virulence $[27,28]$. The iron-sensing regulator Fur controls several traits, including antioxidant defense, siderophore production, and virulence [29]. Recently, we described the regulons of the MarR-type regulators OhrR and EmrR in C. violaceum [30, 31]. We have shown that $\mathrm{OhrR}$ affects $C$. violaceum virulence and is involved in resistance to organic hydroperoxides by regulating ohrA [30,32], while EmrR confers antibiotic resistance by regulating the MFS-type efflux pump Emr$\mathrm{CAB}$ [31]. In this work, we described a novel MarR family transcriptional regulator in C. violaceum, CV_3905, which we named OsbR (oxidative stress response and biofilm regulator). Transcriptome and phenotype analyses revealed that OsbR exerts a global regulatory role in C. violaceum, contributing to oxidative stress defense, anaerobic respiration, and biofilm formation. Our results also indicate a double regulation of OhrR and OsbR on the $o h r R / o h r A$ system for fine-tuning the organic hydroperoxide response.

\section{Results and discussion}

\section{Identification of C. violaceum OsbR (CV_3905) as an OspR/ MgrA homolog}

The MarR-type regulators OspR of P. aeruginosa, MgrA of $S$. aureus, and their homologs in other bacteria, such as AbfR of S. epidermidis and MosR of M. tuberculosis, control multiple and complex bacterial phenotypes [19, $21,23,25]$. To identify the OspR/MgrA homolog in $C$. violaceum, we performed BLASTP analyses with OspR or MgrA against the proteome derived from the genome sequence of C. violaceum ATCC 12472, using default parameters. The search yielded a single hit in C. violaceum, OhrR, which showed 34 and $47 \%$ identity with MgrA and OspR, respectively. Considering that we have previously characterized OhrR in C. violaceum [30, 32], we performed a subsequent search using the PositionSpecific Iteractive BLAST (PSI-BLAST), which uses a position-specific score matrix (PSSM) to found distantly related proteins. In addition to OhrR, this search found as a second hit CV_3905, which shared 30\% identity with MgrA (E value 6e-08 and cover 58\%) and 35\% identity with OspR (E value $2 \mathrm{e}-07$ and cover $52 \%$ ). Based on transcriptome and phenotype characterization presented in this work, we named CV_3905 as OsbR. The OsbR protein harbors two cysteine residues located at positions 55 and 133. Multiple sequence alignment revealed that the Cys55 residue of OsbR is conserved among closely related unstudied proteins. Still, it does not align with the reactive conserved Cys residues of well-characterized redoxsensing OspR/MgrA homologs (Additional file 1, Fig. S1). To characterize OsbR in C. violaceum, we constructed a null mutant strain $\triangle o s b R$ and an overexpressing strain WT $(o s b R)$. The absence of OsbR in $\triangle o s b R$ and the high levels of OsbR in WT(osbR) were confirmed by western 
blot (Fig. 1a). All strains presented the same growth pattern as the WT strain in the LB medium (Fig. 1b).

\section{Transcriptome analyses reveal a large and diverse OsbR regulon}

To identify the OsbR regulon in C. violaceum, we performed two sets of DNA microarray analyses using, in both cases, three biological replicates of mid-log phase bacteria. Comparison of the transcriptome profiles of WT with $\triangle o s b R$ revealed 65 differentially expressed genes (43 downregulated and 21 upregulated genes in $\Delta o s b R$ ) (Additional file 2, Table S1). In comparison, the transcriptome profiles of WT(osbR) versus $\Delta o s b R(\mathrm{pJN} 105)$ showed a higher number of differentially expressed genes (72 downregulated and 121 upregulated genes in $\triangle o s b R$ ) (Additional file 2, Table S2) and more robust differences in the fold-change, including in the genes common to the two datasets (Fig. 2a, Additional file 2, Tables S1 and S2). Interesting, a subset of these genes, including the ohrA/ohrR system (organic hydroperoxide detoxification), the narGHJI operon (nitrate reduction), and the cbaABECF operon (siderophore production) (Fig. 2a, Additional file 2, Table S3), were previously identified as members of the cumene hydroperoxide stimulon in $C$. violaceum [30], indicating a link between OsbR and oxidative stress (see below).

Functional categorization of the up- and downregulated genes identified in both microarray analyses reinforced that OsbR exerts a global transcriptional effect on the C. violaceum transcriptome with an increase in the number of genes and functional processes from the comparison WT versus $\Delta o s b R$ (Fig. $2 \mathrm{~b}$ ) to the comparison WT(osbR) versus $\Delta o s b R(\mathrm{pJN} 105)$ (Fig. 2c). Examples of biological processes affected by OsbR include the categories nitrogen metabolism, membrane transport, iron/siderophore, sulfur and amino acid metabolism, metabolic pathways, regulatory and signaling pathways, among others (Fig. $2 \mathrm{~b}$ and $\mathrm{c}$ ). To validate the microarray data, we performed a Northern blot assay probing the genes narX (narXL operon), narJ (narK1K2GHJI operon), CV_3659 (a MarR family regulator in an operon with cioAB), ohrA (ohrR/ohrA), and CV_0568 (anthranilate synthase in CV_0567-68-69-70 operon) (Fig. 3). The results from both microarrays and the Northern blot assay matched for all genes tested (narX, nar), and CV_3659 were repressed by OsbR while CV_0558 and $o h r A$ were activated by OsbR). The exception was CV_0568 in the comparison WT with $\triangle o s b R$ since it was downregulated in $\triangle o s b R$ in the microarray but not in the Northern blot assay.

\section{OsbR acts directly repressing and activating genes of its regulon}

Several MarR family regulators act as repressors by directly binding in the promoter regions of their target genes $[10,11]$. To verify if OsbR interacts in vitro with its own promoter and the promoter region of OsbR-regulated genes, we purified the His-OsbR protein (Additional file 1, Fig. S2). In the EMSA assays, an increasing amount of His-OsbR was incubated with labeled DNA fragments of the promoter regions (Fig. 4). The DNA binding assay showed that OsbR binds in different concentrations to the promoter regions of osbR, ohrA, CV_2087, narX, and CV_3659. The specificity of these interactions was confirmed by competition assays, adding in the reactions an unspecific $(\mathrm{N})$ or a specific (S) cold probe (Fig. 4, right panels). Interestingly, OsbR binds to the promoter of both repressed (narX and CV_3659) and activated (ohrA, CV_2087) genes, suggesting a dual role of this regulator as repressor and activator. No band-shift was observed to the promoters of narK1, ohrR, cbaF and
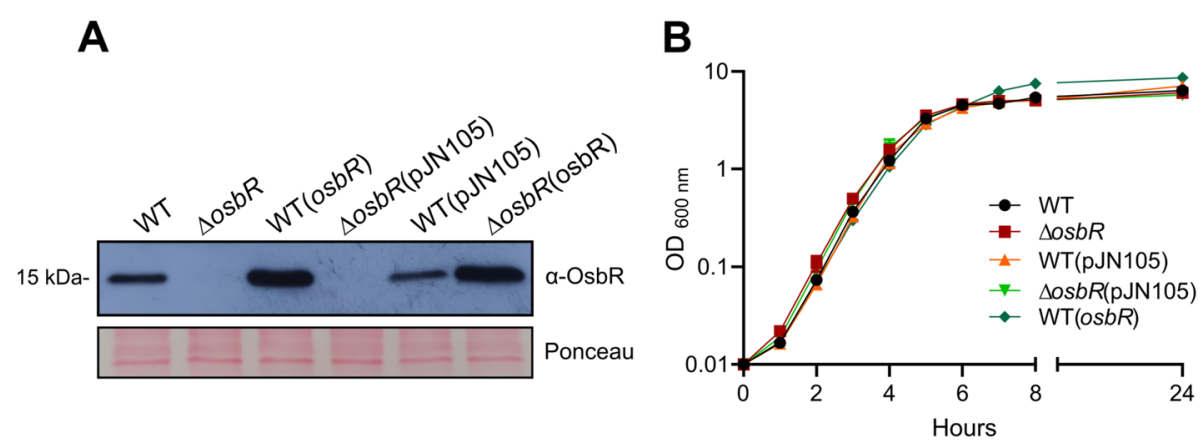

Fig. 1 Construction and analyses of C. violaceum strains with deletion ( $\triangle$ osbR) or overexpression (WT(osbR)) of osbR. a The levels of OsbR in the indicated strains were analyzed by western blot using a polyclonal anti-OsbR antiserum. Equal protein loading was confirmed by Ponceau staining. Full-length blot and Ponceau-stained membrane are presented in Additional file 3, Fig. S3. b Growth curves of the indicated strains in LB medium. All strains showed a similar growth profile 
B



A



C

$\Delta o s b R(p J N 105) \times \mathrm{WT}(o s b R)$

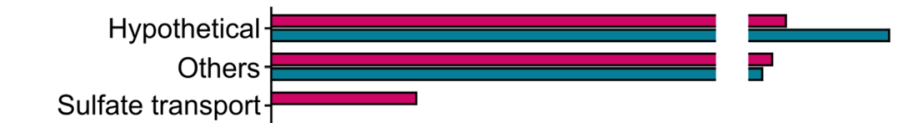

Proteolysic

Energy metabolism

Amino acid transport

Pathogenesis

Siderophore biosynthetic process

Glucose metabolic process

Regulation of transcription

Response to oxidative stress

Amino acid metabolic process

Nitrate assimilation/metabolic process

Signal transduction

Carbohydrate transport/metabolic process

Aerobic respiration

Fatty acid biosynthetic/metabolic process

Transmembrane/Protein transport

Biosynthetic process

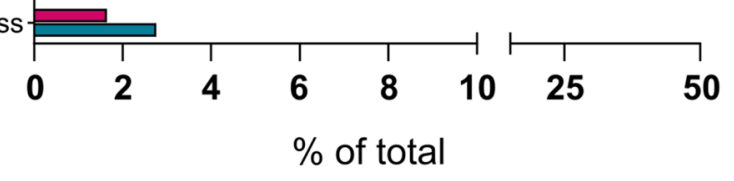

Fig. 2 Microarray analyses revealed that ObsR regulates many genes involved in several biological processes. a Venn diagrams comparing the differentially expressed genes from the microarray analyses of this work (osbR strains) with published data (WT treated with the oxidant CHP). b, c Functional categorization by Gene Ontology (GO) of the differentially expressed genes from the comparison wild-type versus $\triangle o s b R$ (b) and WT(osbR) versus $\triangle$ osbR(pJN105) (c)

to the negative control (the coding region of CV_0208) (Fig. 4), indicating that these genes are indirectly regulated by OsbR. Considering that many transcriptional regulators are members of the OsbR regulon (Fig. 2c, Additional file 2, Table S2), it is tempting to propose that OsbR has its global regulatory role amplified by regulating other transcriptional regulators.

\section{OsbR responds to oxidation and has an antioxidant role} Considering that OsbR has two cysteine residues (Additional file 1, Fig. S1) and when overexpressed activates the genes ohrA/ohrR (Fig. 3, Fig. 4, Additional file 2, Table S2), which encode an organic hydroperoxide antioxidant system in C. violaceum [30, 32], we investigated whether OsbR dimerizes upon oxidation by 


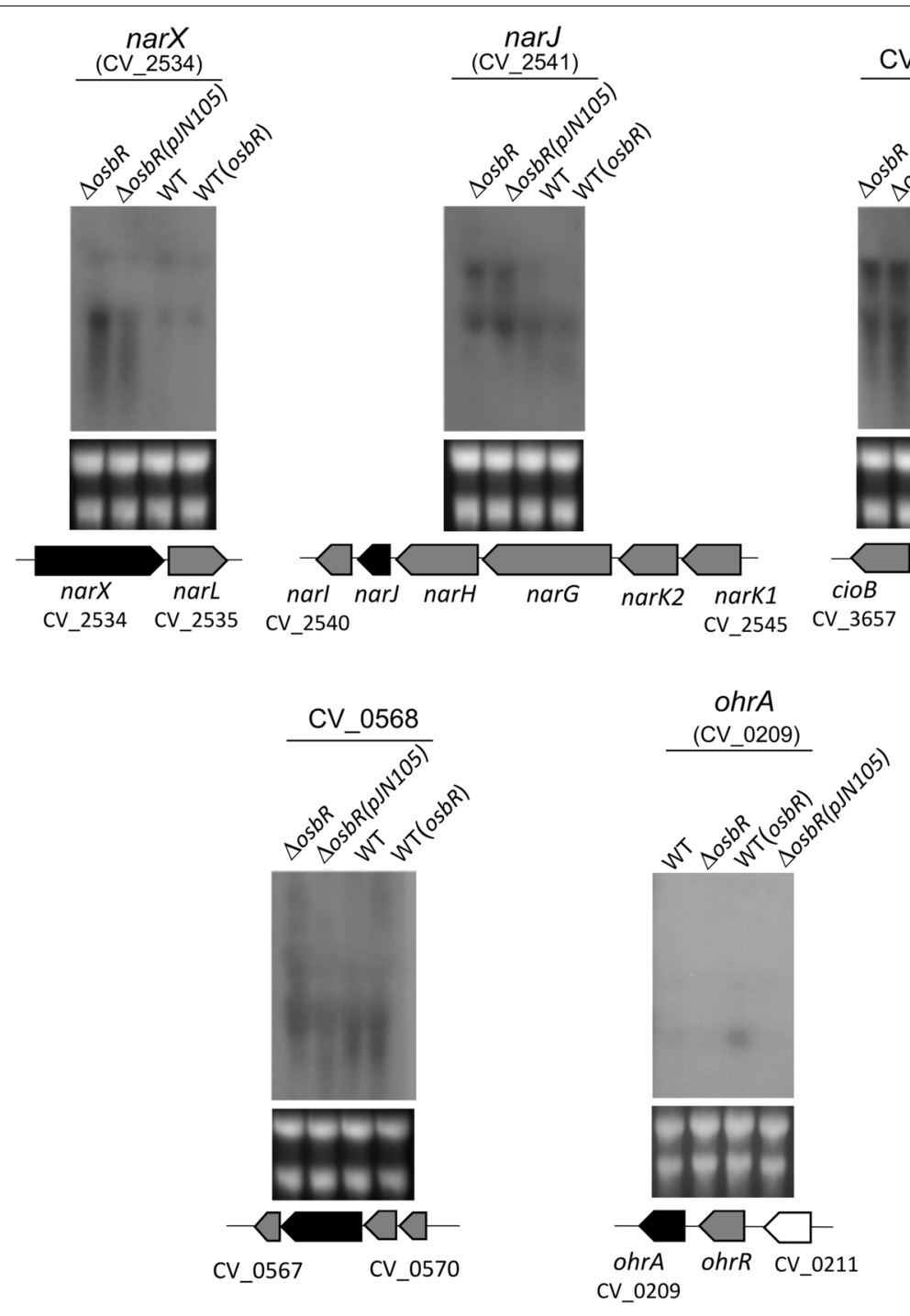

Fig. 3 Validation by Northern blot of selected genes regulated by OsbR. RNA samples from the indicated C. violaceum strains (the same strains used in the microarray analyses) were transferred to a membrane and hybridized with specific radiolabeled probes for the selected genes (indicated by black arrows in the gene maps). The selected genes were repressed (narX, narJ, and CV_3659) or activated (CV_0568 and ohrA) by OsbR. Grey arrows indicate neighbor genes also differentially expressed according to microarray analyses. The rRNA were used as a loading control (Bottom gels). Full-length blot and RNA loading gel are presented in Additional file 3, Fig. S4

intermolecular disulfide bond formation, a feature of many Cys-based redox sensing regulators $[16,17,19,23$, 32]. Indeed, the dimerization of monomeric purified HisOsbR was verified in non-reducing SDS-PAGE gel when this protein was incubated with the oxidants tert-butyl hydroperoxide (TBHP), cumene hydroperoxide (CHP), and hydrogen peroxide $\left(\mathrm{H}_{2} \mathrm{O}_{2}\right)$ at 0.1 and $1 \mathrm{mM}$, but not with the reductant agent dithiothreitol (DTT) (Fig. 5a). Accordingly, when pretreated with $\mathrm{CHP}$, the oxidized OsbR did not bind to the promoter regions of ohrA, CV_2087, CV_3659, narX, and osbR (Fig. 5b). Although further investigation is required to define the role of the
OsbR Cys residues on oxidation sensing, our data indicated that the DNA binding activity of OsbR is inhibited by its oxidation.

Next, we investigated the relationship between OsbR and the OhrA/OhrR system on the response to organic hydroperoxides by Northern blot and growth curve assays (Fig. 6). The ohrA expression was increased in the wild-type strain under CHP treatment and reached the maximum levels even without treatment in the $\Delta o h r R$ mutant (Fig. 6a), confirming our previous data that $\mathrm{OhrR}$ is a repressor of ohrA [30, 32]. Importantly, deletion of $o s b R$ in both the wild-type and $\Delta o h r R$ 




backgrounds decreased the ohrA expression under CHP treatment (Fig. 6a), suggesting that the maximum ohrA expression requires both OhrR derepression and OsbR activation. Indeed, overexpression of OsbR caused activation of ohrA even in the absence of oxidative stress (Fig. 3). Consistently with this regulatory model, the growth curves under treatment with the oxidants TBHP (Fig. 6b) and CHP (Fig. 6c) indicated that the strains with high $o h r A$ levels (WT(osbR), $\Delta o h r R(o s b R)$, and $\Delta o h r R)$ showed better growth than the wild-type and $\triangle o s p R$ strains. Our results showing an interconnection of OhrR and OsbR on the regulation of ohrA in C. violaceum resemble the cross-regulation of OhrR and OspR on the ohr and $g p x$ genes described in $P$. aeruginosa [18]. The OspR of $P$. aeruginosa is a homolog of OhrR that binds and represses ohr and can even functionally complement an ohrR mutant strain [18]. Despite OspR being a repressor of ohr and OsbR a putative activator of $o h r A$, both transcriptional regulators are responsive to organic hydroperoxides.

Among the genes upregulated by CHP oxidative stress and activated by OsbR overexpression (Fig. 2a, Additional file 2, Table S3), we highlight the cbaABECF operon in addition to the ohrA and ohrR genes. Recently, we determined that this operon encodes enzymes required for the production of a catecholatetype siderophore in C. violaceum [33]. The E. coli catecholate-type siderophore enterobactin has recently been shown to be involved in protection against oxidative stress via an iron-independent radical scavenging activity [34]. The fact that the cbaABECF operon is activated by OsbR like ohrA in C. violaceum could suggest a dual OsbR-mediated mechanism for protection against oxidative stress. 


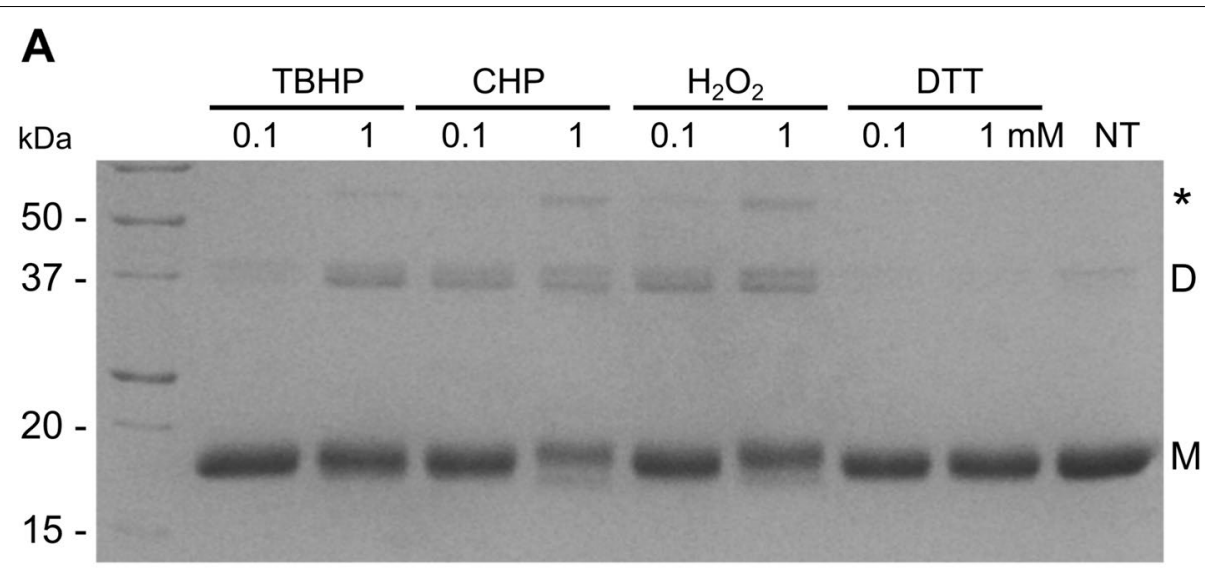

B

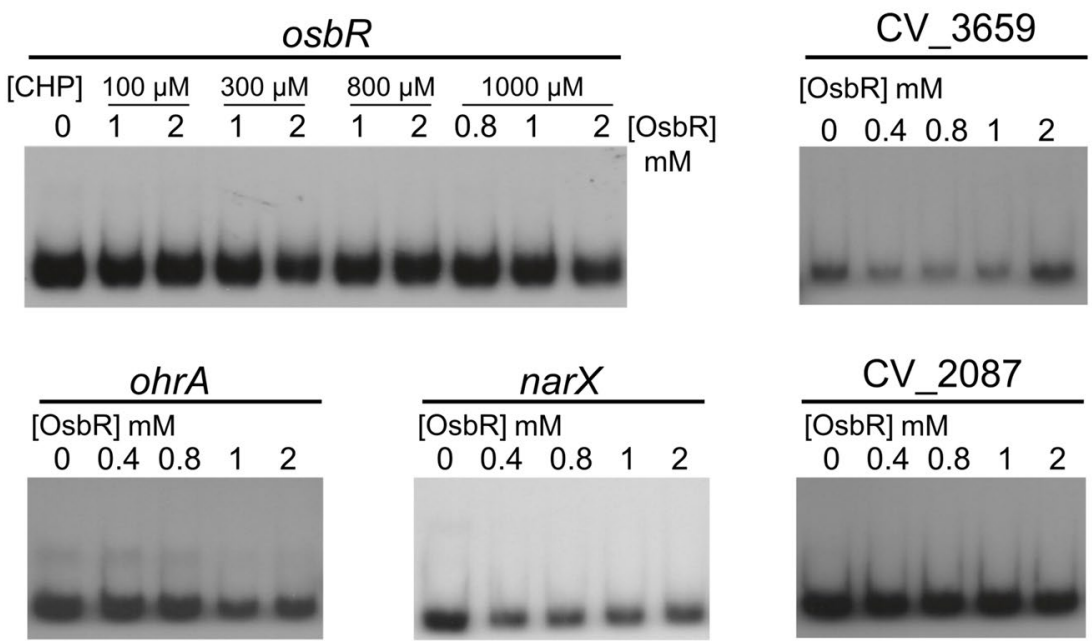

Fig. 5 OsbR oxidation causes dimerization and inhibition of its DNA binding activity. a Formation of covalent OsbR dimers under oxidative stress in vitro. After 2 hours of exposure to oxidants or DTT, the His-OsbR protein was analyzed by non-reducing SDS-PAGE. NT refers to nontreated protein. Monomeric and dimeric forms are indicated by $M$ and D, respectively. Asterisk indicates a third unknown multimeric form. $\mathbf{b}$ EMSA assay with oxidized OsbR. Prior to incubation with the DNA probes, His-OsbR was oxidized with $1 \mathrm{mM}$ CHP for 1 hour (for the osbR probe, OsbR was oxidized with the indicated CHP concentrations)

\section{OsbR regulates genes involved in anaerobic nitrate respiration in C. violaceum}

Among the differentially expressed genes found in both microarray analyses and showing the highest upregulation in the $\triangle o s b R$ mutant were those involved in nitrate respiration (Fig. 2, Additional file 2, Tables S1 and S2). These genes include the narXL operon encoding for a two-component system (NarXL) and the narK$1 K 2$ GHJI operon encoding for nitrate/nitrite transporters (NarK1 and NarK2) and a respiratory nitrate reductase (NarGHJI). The OsbR-mediated repression of both operons was validated by Northern blot (Fig. 3). As the OsbR binding occurred in the promoter of the narXL operon but not in the promoter of narK1K2GHJI (Fig. 4), we propose that OsbR acts via NarXL to regulate the narK1K2GHJI operon in C. violaceum. Indeed, the NarXL is a well-characterized two-component system that senses the nitrate/nitrite levels to activate narGHJI and narK expression in Escherichia coli and Pseudomonas stutzeri $[35,36]$. Consistently with our data, it has been demonstrated that other MgrA/OspR homologs, such as MosR in M. tuberculosis [25, 37] and RosR in Corynebacterium glutamicum [38] regulate nar genes associated with the nitrate reduction for growth under hypoxic and anaerobic conditions [39].

Based on genome prediction [5, 6] and experimental data [40], C. violaceum can undergo anaerobic respiration using nitrate as an electron acceptor. Considering the remarkable role of OsbR on repressing the nar genes, we checked the involvement of $o s b R$ 




in anaerobic respiration, growing the $C$. violaceum strains in LB medium in an anaerobic jar (Fig. 7). No bacterial growth occurred in LB unless this medium was supplemented with $\mathrm{NaNO}_{3}$, confirming that $C$. violaceum uses nitrate to obtain energy in anaerobic conditions. When compared with the WT strain, the $\Delta o s b R$ strain had an impaired growth, while the $\mathrm{WT}(o s b R)$ strain showed an improved growth (Fig. 7). These data indicate that the overexpression of the nar genes (in $\triangle o s b R$ ) was detrimental while their repression (in WT $(o s b R)$ ) was beneficial under these conditions. Although $C$. violaceum possesses the enzymes nitrate reductase (Nar), nitrite reductase (Nir), and nitric oxide reductase (Nor) allowing denitrification until nitrous oxide $\left(\mathrm{N}_{2} \mathrm{O}\right)[5,6,40]$, OsbR affected the expression only of the nar genes. Therefore, the elevated expression of the nar genes could enhance the reduction of nitrate to nitrite in the $o s b R$ mutant, causing nitrite accumulation and toxicity. Nitrite accumulation is a challenge in bacteria that lack nitrite reductase $[41,42]$. Otherwise, in $P$. aeruginosa, a strain overexpressing the response regulator AtvR had upregulation of all denitrification genes (nar, nir, nor, and nos operons) and improved growth coupled to anaerobic nitrate reduction [43].

\section{OsbR represses biofilm formation in C. violaceum}

Several MarR-type regulators homologs to OspR/MgrA have been associated with biofilm formation, including MgrA and SarZ of S. aureus [21, 44, 45], AbfR of S. epidermidis [23], and AsrR of E. faecium [26]. To investigate whether OsbR affects biofilm formation in C. violaceum, we performed a biofilm assay with cultures grown statically in LB medium. Deletion of $o s b R$ had no effect, while overexpression of $o s b R$ caused a pronounced reduction in the biofilm formation (Fig. 8). Detailed inspection of our 


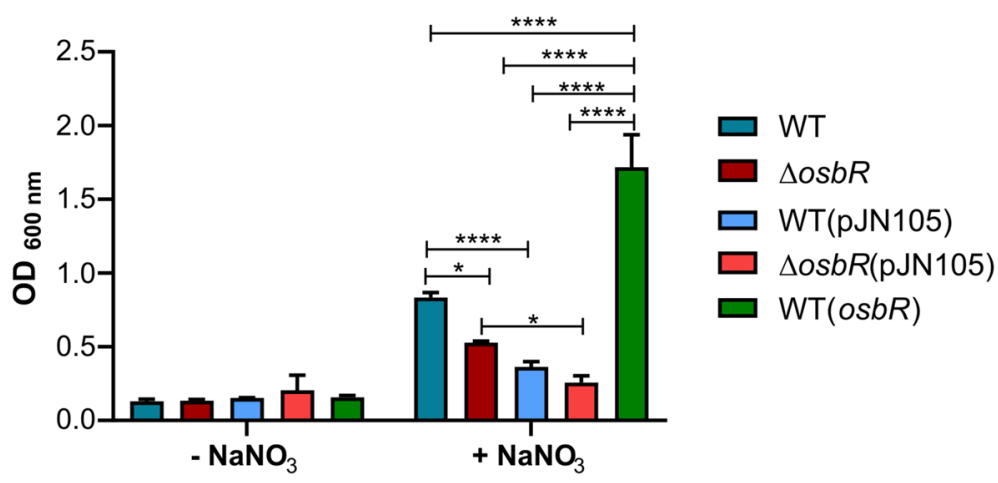

Fig. 7 OsbR affects C. violaceum growth under anaerobic conditions. The indicated strains of $C$. violaceum were diluted to $\mathrm{OD}_{600}=0.1$ in $L B$ medium or $\mathrm{LB}$ plus $0.5 \% \mathrm{NaNO}_{3}$. The cultures were grown without agitation in an anaerobic jar and the $\mathrm{OD}_{600}$ was measured after $48 \mathrm{~h}$. Statistical analysis was performed by Two-way ANOVA and $P$ value $<0.05$ was considered significant. $P$ value $<0.05={ }^{*} ; P$ value $<0.01={ }^{* *} ; P$ value $<0.001={ }^{* * *} ; P$ value $<0.0001{ }^{* * * *}$

microarray data indicated that genes from two clusters encoding putative type I secretion systems and related RTX adhesins (CV_1734-35-36-37 and CV_0513-15-16) were upregulated in the absence of OsbR when comparing WT( $o s b R)$ with $\Delta o s b R(\mathrm{pJN} 105)$ (Additional file 2, Table S2). As adhesins are important to surface attachment and biofilm development [46], we propose that the $o s b R$ overexpression caused a reduction in the biofilm formation owing to the OsbR-mediated repression of these two gene clusters. However, more work will be needed to prove this assumption and to define the role of RTX proteins in C. violaceum. In agreement with our data, in S. aureus and E. faecium, the regulators MgrA and AsrR negatively regulate biofilm production by repressing several surface adhesins $[21,26,44]$.

It will be of interest in future studies to address whether OsbR senses oxidants via its cysteine residues to regulate its target genes. Moreover, genetic epistasis analyses could provide a link of the phenotypes related to OsbR (oxidative stress, biofilm formation, and anaerobic growth) and the specific genes of the OsbR regulon.

\section{Conclusions}

In this work, we presented transcriptome and phenotypic characterization of OsbR, a yet unstudied MarR family transcriptional regulator of C. violaceum. We
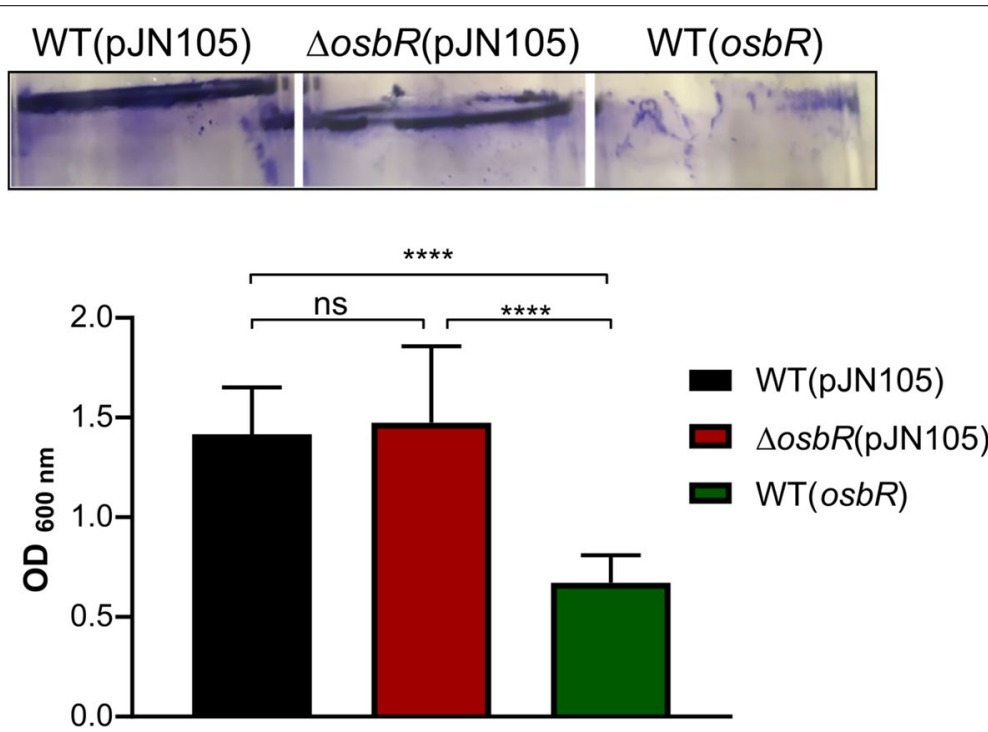

Fig. 8 OsbR represses biofilm formation in C. violaceum. Strains were diluted to $\mathrm{OD}_{600}=0.01$ in $\mathrm{LB}$ medium, and the biofilm formation was measured after growth in static condition for $24 \mathrm{~h}$. Upper: Representative test tubes for each strain after biofilm staining with violet crystal. Bottom: Quantification of biofilm formation by measurement of $\mathrm{OD}_{600}$. Statistical analysis was performed with One-way ANOVA with $P$ value $<0.05$ being considered significant. ${ }^{* * *}, P$ value $<0.0001$ 
showed that OsbR regulates many genes involved in diverse cellular functions as its counterparts OspR/ MgrA. Among the OsbR-regulated genes, we highlighted those encoding proteins associated with organic hydroperoxide detoxification (ohrA/ohrR), nitrate assimilation (narK1K2), anaerobic nitrate respiration (narGHJI), and biofilm formation (putative type I secretion systems and associated adhesins). Phenotypic assays using C. violaceum strains harboring null mutation or overexpression of osbR confirmed the importance of OsbR to organic hydroperoxide resistance (probably by OsbR activation of $o h r A$ ), anaerobe nitrate respiration (by strong repression of nar operons), and biofilm formation (by repression of adhesins). The EMSA assays revealed that reduced but not oxidized OsbR binds to the promoter regions of either repressed or activated genes. Together, the results outline the global function and importance of OsbR as a novel MarR-type regulator in C. violaceum.

\section{Methods}

\section{Bacterial strains and growth conditions}

All the bacterial strains and plasmids used in this work are listed in Table 1 . The Chromobacterium violaceum wild-type (WT) strain ATCC 12472 and its derivative strains were grown at $37^{\circ} \mathrm{C}$ in Luria-Bertani (LB) medium supplemented with gentamycin $(40 \mu \mathrm{g} / \mathrm{ml})$ or kanamycin $(50 \mu \mathrm{g} / \mathrm{ml})$, when necessary. Mutant strains harboring an in-frame deletion of $o s b R(\triangle o s b R)$ and of $o s b R$ and $o h r R(\Delta o s b R \Delta o h r R)$ were constructed by homologous recombination, using a previously described allelic exchange protocol based on sucrose selection [29, 31-33]. All mutant strains were confirmed by PCR and DNA sequencing (data not shown). To obtain an $o s b R$ overexpressing strain (WT $(o s b R)$ ), the $o s b R$ gene with its own promoter was cloned into the pJN105 plasmid, and the resulting construct was introduced in the WT strain by conjugation. DNA cloning was performed by digestion with restriction enzymes of PCR-amplified DNA products (Table 2).

\section{Growth curves}

Growth curves of the C. violaceum strains were performed in $\mathrm{LB}$ medium at $37^{\circ} \mathrm{C}$ and $250 \mathrm{rpm}$ agitation in Erlenmeyer flasks. Overnight liquid cultures were diluted to an optical density at $600 \mathrm{~nm}\left(\mathrm{OD}_{600}\right)$ of 0.01 . Samples were collected at the indicated time points to measure the $\mathrm{OD}_{600}$ in a BioPhotometer (Eppendorf). The growth curves under oxidant conditions were performed in 96-well plates. We added $120 \mu \mathrm{M}$ tert-butyl hydroperoxide (TBHP) or $55 \mu \mathrm{M}$ cumene hydroperoxide (CHP) to the C. violaceum $\mathrm{LB}$ cultures at $\mathrm{OD}_{600}$ of 0.01 . The growth was determined by measuring for $24 \mathrm{~h}$ the $\mathrm{OD}_{600}$ at 15 min intervals in a SpectraMax i3 MiniMax Imaging

Table 1 Bacterial strains and plasmids

\begin{tabular}{|c|c|c|}
\hline Strain or plasmid & Description & Reference or source \\
\hline \multicolumn{3}{|l|}{ Escherichia coli strains } \\
\hline $\mathrm{DH} 5 \mathrm{a}$ & Strain for cloning & {$[47]$} \\
\hline S17-1 & Strain for plasmid mobilization & {$[48]$} \\
\hline BL21(DE3) & Strain for protein expression & Novagen \\
\hline \multicolumn{3}{|c|}{ Chromobacterium violaceum strains } \\
\hline ATCC 12472 & Wild-type strain (Sequenced genome) & {$[6]$} \\
\hline JF3905 & Wild-type strain with the CV_3905 gene deleted ( $\triangle$ osbR) & This work \\
\hline JF0210 & Wild-type strain with the CV_0210 gene deleted ( $\triangle$ ohrR) & {$[32]$} \\
\hline JF39050210 & Wild-type strain with the CV_3905 and CV_0210 genes deleted ( $\triangle$ osbR $\triangle$ ohrR) & This work \\
\hline WT(pJN105) & Wild-type strain containing the vector pJN105 empty & This work \\
\hline WT(osbR) & Wild-type strain overexpressing osbR & This work \\
\hline$\triangle 0 s b R(p J N 105)$ & Mutant osbR containing the vector pJN105 empty & This work \\
\hline$\triangle o s b R(o s b R)$ & Mutant osbR strain overexpressing osbR & This work \\
\hline \multicolumn{3}{|l|}{ Plasmids } \\
\hline pNPTS138 & Suicide vector containing oriT $\operatorname{sac} B$; Kanamycin resistance & D. Alley \\
\hline pJN105 & Broad-host-range vector; Gentamycin resistance & [49] \\
\hline pET15b & His-tagged protein expression vector; Ampicillin resistance & Novagen \\
\hline pNPTS138 $\triangle 0 s b R$ & In frame null deletion of osb $R$ & This work \\
\hline pNPTS138 $\triangle$ ohrR & In frame null deletion of ohrR & [32] \\
\hline pJN105 osbR & Overexpression of osbR & This work \\
\hline pET15b osbR & Heterologous expression of the His-OsbR protein & This work \\
\hline
\end{tabular}


Table 2 Primers used in this work

\begin{tabular}{|c|c|}
\hline Primer & Sequence $\left(5^{\prime}->3^{\prime}\right)$ \\
\hline \multicolumn{2}{|c|}{ Mutant and overexpressing strains } \\
\hline CV3905-del1 & TAGGGCCCGAAACTGCGGCTCGAACGCC \\
\hline CV3905-del2 & ATCAAGCTTCATCGAAAGAGTCCCCGTGC \\
\hline CV3905-del3 & ATCAAGCTTGAAAACACGCCGGCCTGAGC \\
\hline CV3905-del4 & TTGGATCCCTGGTGGCGGGCTTTGTTGC \\
\hline CV_3905-Comp-Fw & GGTACCCTGCAGAGCCAGTCCGCATTAAGGCG \\
\hline CV_3905-Sup-Rv & GGTTACGAGCTCGACGCTCAGCGTCAGAGCTG \\
\hline \multicolumn{2}{|l|}{ Northern blot } \\
\hline CV0209-NB-Fw & TGCAGGTGAAGTTGAGCACCCC \\
\hline CV0209-NB-RV & CCGATGAAGCAGGCGGAATAGCC \\
\hline NB CV2534 -FW & GATGGTGCTGATCGCGATGG \\
\hline NB CV2534-Rv & CCAGGGTCTCGATCAGCATC \\
\hline NB CV2541-Fw & CCATTCTGTCCGCGCTGTTG \\
\hline NB CV2541-Rv & GCATTTCGTCCATGTCGCGG \\
\hline NB CV3659-Fw & CACCTCTGGTGCAGGCTTTC \\
\hline NB CV3659-RV & GACAGCGACTGGGTGAACTG \\
\hline NB CV0568-Fw & ACGCCAAGCTGGGACTCAAC \\
\hline NB CV0568-Rv & GCTCTACCTCCCGTTCGATG \\
\hline \multicolumn{2}{|l|}{ EMSA } \\
\hline pCV0209-Fw & TTGGATCCTGCCGGAGCGGAATGCAACT \\
\hline pCV0209-Rv & ATTACTGCAGGCGGTGGCTTCGGCGGTATAC \\
\hline pCV3905-Fw & GGTACCCTGCAGAGCCAGTCCGCATTAAGGCG \\
\hline pCV3905-Rv & ATCAAGCTTCATCGAAAGAGTCCCCGT \\
\hline pCV0210-Fw & CTCGCTGGCCGATCTGGGAC \\
\hline pCV0210-Rv & GGCGGAATACAGCGCGAAGC \\
\hline pCV1486Fw & GACCAAGTGATAGACGCCGG \\
\hline CV1486-DEL2 & GGCCTAAAGCTTCTCGGTCATGAGGATGTCTG \\
\hline pCV2087Fw & CCTCGTAAAAGCGGATGGCG \\
\hline pCV2087Rv & CGTCGAAACCCTTCATCGCG \\
\hline pCV2534Fw & GATCACCGACACCCTCAAGC \\
\hline pCV2534RV & GCGACAACACCAGCAGCTTG \\
\hline pCV2545Fw & TGACTCTCCCCCATTGGGAG \\
\hline pCV2545Rv & ATCGGGATGCCCAGCACCGC \\
\hline pCV3659Fw & GACCGACAATGACGGCTACG \\
\hline pCV3659Rv & GCCGAAATGCAGCACGAAAG \\
\hline CV0208 FW & GACCATACTAGACAGCTACGCC \\
\hline CV0208Rv & CAGCGTGGTGATTTGCGGATAG \\
\hline
\end{tabular}

Underlined letters indicate restriction enzyme recognition sites used for cloning purposes.

Cytometer. Data were plotted in one-hour intervals for clarity.

\section{Growth under anaerobic conditions}

Overnight cultures of $C$. violaceum were diluted in $2 \mathrm{~mL}$ of $\mathrm{LB}$ to $\mathrm{OD}_{600 \mathrm{~nm}}$ of 0.1 . Cultures were grown in LB or LB plus $5 \% \mathrm{NaNO}_{3}$ without agitation at $37^{\circ} \mathrm{C}$ in an anaerobic jar with a GasPak EZ Anaerobe Container System (catalog number 260001; Becton Dickinson) to establish anaerobic conditions. The growth was determined by measuring the $\mathrm{OD}_{600}$ after cultivation by $48 \mathrm{~h}$.

\section{Biofilm formation}

Biofilm was quantified using a crystal violet method as previously described [29]. Briefly, C. violaceum liquid cultures at an $\mathrm{OD}_{600}$ of 0.01 were grown without agitation at $37^{\circ} \mathrm{C}$ for $24 \mathrm{~h}$ in $1.5 \mathrm{~mL}$ LB in glass tubes. The biofilm was stained with crystal violet, solubilized with ethanol, and quantified by measuring the $\mathrm{OD}_{600}$. 


\section{RNA isolation}

The C. violaceum strains in three biological replicates (for DNA microarray assays) were grown in LB medium at $37^{\circ} \mathrm{C}$ and $250 \mathrm{rpm}$ agitation until mid-log phase $\left(\mathrm{OD}_{600}\right.$ of 0.8 to 1.2$)$. The cultures were centrifugated, and the total RNA was extracted with the TRIzol reagent (Ambion) and purified with the illustra RNAspin Mini RNA isolation kit (GE Healthcare). The RNA integrity was evaluated by formaldehyde agarose gel. The absence of DNA contamination was confirmed by PCR. Quantification of total RNA was measured with a NanoDrop spectrophotometer (Thermo Scientific).

\section{DNA microarray}

The procedures for cRNA labeling, hybridization, and washing were performed according to the Agilent TwoColor Microarray-Based Exon Analysis protocol (Agilent Technologies). Briefly, $100 \mathrm{ng}$ RNA of each sample were converted in cRNA and labeled with Cy3 and Cy5 using the Low Input Quick Amp WT Two-Color kit (Agilent Technologies). Equimolar amounts of oppositely labeled cRNA were hybridized at $65^{\circ} \mathrm{C}$ by $17 \mathrm{~h}$ in a customdesigned oligonucleotide microarray slide [30]. The slides were scanned with an Agilent High-Resolution C Scanner. The data were extracted and normalized by using Agilent Feature Extraction Image Analysis Software (Version 10.7.3). Data analysis to determine the differentially expressed genes was performed as previously described $[30,31]$. Briefly, the values for the relative expression of each gene were calculated as the average of the values of all probes corresponding to the same gene (ratio values of at least 27 probes, 9 from each biological replicate). Statistical significance was calculated according to the chi square $p$-value compositional method [50], as implemented in [51]. Briefly, the log-ratio $p$-values for each probe were obtained from Agilent Feature Extraction Software. The global significance level was controlled at 0.05 using the highly stringent Bonferroni multiple correction method. Considering that multiple hypothesis testing were performed $(45,220 * 3=135,660$, number of probes in biological triplicates), the effective cutoff is $0.05 / 135660=3.68^{*} 10^{-7}$. Genes were considered differentially expressed if showed simultaneously (i) 2-fold change and (ii) statistical significance at 0.05 $\left(<3.68 * 10^{-7}\right)$.

\section{Northern blot}

For the microarray validation, we used $7.5 \mu \mathrm{g}$ of the same RNA samples. For the expression of ohrA under oxidant condition, we used $5 \mu \mathrm{g}$ of total RNA extracted from the WT, $\Delta o s b R, \Delta o h r R$, and $\Delta o s b R \Delta o h r R$ strains grown in LB untreated or treated with $100 \mu \mathrm{M}$ of cumene hydroperoxide (CHP) for $10 \mathrm{~min}$. After running in denaturing formaldehyde agarose gel, the RNA samples were transferred onto nylon Hybond-XL membranes (GE Healthcare). Specific probes for the indicated genes were generated by PCR (Table 2 ) and radiolabeled with $\left[\alpha^{32} \mathrm{P}\right]$ $\mathrm{dCTP}$ according to the DECAprime kit (Ambion) protocol. RNA transfer, membrane hybridization and washing, and signal detection were performed as described [30, 32]. Briefly, RNA was transferred to membranes by capillarity. Membranes were prehybridized and incubated with the labeled probes in $10 \mathrm{ml}$ of ULTRAhyb (Ambion). Washing was performed according to the manufacturer's instructions and as previously described [30].

\section{Expression and purification of his-OsbR}

The osbR gene (CV_3905) was PCR-amplified and cloned into the expression vector pET15b (Table 2). The E. coli BL21(DE3) harboring the plasmid pET15b-osbR was used to express the recombinant His-tagged OsbR protein after induction with $1 \mathrm{mM}$ IPTG for $2 \mathrm{~h}$ at $37^{\circ} \mathrm{C}$. The His-OsbR protein was purified by affinity chromatography in a Ni-NTA superflow column (Qiagen) as previously described [32].

\section{OsbR dimerization in vitro}

The purified His-OsbR protein was incubated with the reductant agent dithiothreitol (DTT) or the oxidants cumene hydroperoxide (CHP), tert-butyl hydroperoxide (TBHP), and hydrogen peroxide $\left(\mathrm{H}_{2} \mathrm{O}_{2}\right)$ at concentrations of 0.1 and $1 \mathrm{mM}$ for $30 \mathrm{~min}$ at room temperature. The samples were alkylated with $100 \mathrm{mMN}$-ethylmaleimide (NEM) for $2 \mathrm{~h}$. The presence of OsbR as monomers or dimers was analyzed after non-reducing SDS-PAGE and Coomassie brilliant blue staining.

\section{Electrophoretic mobility shift assay (EMSA)}

DNA probes corresponding to the promoter region of the indicated genes and the coding region of CV_0208 were amplified by PCR with specific primers (Table 2). These fragments were labeled with $\left[\gamma^{32} \mathrm{P}\right]$-ATP by T4 polynucleotide kinase (Thermo Scientific) and purified with the Wizard SV gel and PCR clean-up system (Promega). The interaction of reduced OsbR with the DNA probes was performed as already described [30,32]. The EMSA with oxidized OsbR was performed by pretreatment of OsbR with $1 \mathrm{mM} \mathrm{CHP} \mathrm{by} 1 \mathrm{~h}$ at room temperature.

\section{Western blot}

The C. violaceum strains were grown in LB until the midlog phase. Samples were collected, centrifugated and the pellets were resuspended in an SDS sample buffer. Proteins were resolved by $15 \%$ SDS-PAGE and transferred onto a nitrocellulose membrane. The western blot was performed with the Protein Detector LumiGLO 
Western blot (KPL) kit, using an anti-OsbR polyclonal antiserum (1:1000) developed in mice by a subcutaneous injection of the His-OsbR protein. The six-weekold female BALB/c mice $(n=5)$ used in this study were obtained from the facility Biotério Geral (PUSP-RP), and maintained in the Animal Facilities of the Faculdade de Medicina de Ribeirão Preto (FMRP-USP). The animals were euthanized with an excessive dose of anesthetic (one dose of $200 \mathrm{mg} / \mathrm{kg}$ ketamine and $20 \mathrm{mg} / \mathrm{kg}$ xylazine).

\section{Supplementary Information}

The online version contains supplementary material available at https://doi. org/10.1186/s12866-021-02369-x.

Additional file 1: Figure S1 OsbR alignment. Figure S2 His-OsbR purification by affinity chromatography.

Additional file 2: Table S1 Comparison of the transcriptome profiles of WT with $\triangle O s b R$. Table S2 Comparison of the transcriptome profiles of WT(osbR) versus $\triangle o s b R(p J N 105)$. Table S3 List of genes shared among our microarray analyses and the CHP stimulon.

Additional file 3: Figure S3 Uncropped blot. Figure S4 Uncropped Northen blot. Figure S5 Uncropped Northern blot.

\section{Acknowledgments}

We are grateful to the Sergio L Salvador lab for assistance with the anaerobic growth assays and the Geraldo A Passos lab for assistance with the microarray scanning.

\section{Authors' contributions}

JAA and JFSN planned the experiments and wrote the manuscript; JAA, MPM, and KCMM performed the experimental work; JAA, MPM, and JFSN analyzed and interpreted data; TK performed the statistical analysis of the microarray data; JFSN participated in study coordination and funding acquisition. All authors read and approved the final manuscript.

\section{Funding}

This research was supported by grants from the São Paulo Research Foundation (FAPESP; grants 2012/20435-9 and 2020/00259-8) and Fundação de Apoio ao Ensino, Pesquisa e Assistência do Hospital das Clínicas da FMRP-USP (FAEPA). During the course of this work, JAA (grant 2016/08728-1), MPM (grant 2013/25745-9), and KCMB (grant 2013/18797-2) were supported by FAPESP fellowships. The funders had no role in the design of the study, collection, analysis, and interpretation of data, and in writing the manuscript.

\section{Availability of data and materials}

The data supporting the conclusions of this article are included within the article and its additional files. The microarray datasets are available in the Gene Expression Omnibus (GEO) database (https://www.ncbi.nlm.nih.gov/geo/) with the accession number GSE171860.

\section{Declarations}

\section{Ethics approval and consent to participate}

The experiments with mice (antibody production) were performed following the Ethical Principles in Animal Research adopted by the National Council for the Control of Animal Experimentation (CONCEA), and the protocol was approved by the Local Ethical Animal Committee (CEUA) from FMRP-USP (number 147/2014). The study was carried out in compliance with the ARRIVE guidelines.

\section{Consent for publication}

Not applicable.

\section{Competing interests}

The authors declare that they have no competing interests.

\section{Author details}

${ }^{1}$ Departamento de Biologia Celular e Molecular e Bioagentes Patogênicos, Faculdade de Medicina de Ribeirão Preto, Universidade de São Paulo, Ribeirão Preto, SP, Brazil. ${ }^{2}$ Departamento de Bioquímica e Imunologia, Faculdade de Medicina de Ribeirão Preto, Universidade de São Paulo, Ribeirão Preto, SP, Brazil.

Received: 15 April 2021 Accepted: 26 October 2021

Published online: 04 November 2021

\section{References}

1. Durán N, Menck CFM. Chromobacterium violaceum: a review of pharmacological and industrial perspectives. Crit Rev Microbiol. 2001;27(3):201-22.

2. Batista JH, da Siva Neto JF. Chromobacterium violaceum pathogenicity: updates and insights from genome sequencing of novel Chromobacterium species. Front Microbiol. 2017;8:2213.

3. Durán N, Justo GZ, Ferreira CV, Melo PS, Cordi L, Martins D. Violacein: properties and biological activities. Biotechnol Appl Biochem. 2007;48(Pt 3):127-33.

4. Yang $\mathrm{CH}, \mathrm{Li} \mathrm{YH}$. Chromobacterium violaceum infection: a clinical review of an important but neglected infection. J Chinese Med Assoc. 2011;74(10):435-41.

5. Creczynski-Pasa TB, Antônio RV. Energetic metabolism of Chromobacterium violaceum. Genet Mol Res. 2004;3(1):162-6.

6. Vasconcelos ATR, Almeida DF, Hungria M, Guimarães CT, Antônio RV, Almeida FC, et al. The complete genome sequence of Chromobacterium violaceum reveals remarkable and exploitable bacterial adaptability. Proc Natl Acad Sci U S A. 2003;100(20):11660-5.

7. Hungria M, Nicolás MF, Guimarães CT, Jardim SN, Gomes EA, De Vasconcelos ATR. Tolerance to stress and environmental adaptability of Chromobacterium violaceum. Genet Mol Res. 2004;3(1):102-16.

8. Cohen SP, Hachler H, Levy SB. Genetic and functional analysis of the multiple antibiotic resistance (mar) locus in Escherichia coli. J Bacteriol. 1993;175(5):1484-92.

9. Ellison DW, Miller VL. Regulation of virulence by members of the MarR/ SlyA family. Curr Opin Microbiol. 2006;9(2):153-9.

10. Wilkinson SP, Grove A. Ligand-responsive transcriptional regulation by members of the MarR family of winged helix proteins. Curr Issues Mol Biol. 2006;8(1):51-62.

11. Perera IC, Grove A. Molecular mechanisms of ligand-mediated attenuation of DNA binding by MarR family transcriptional regulators. J Mol Cell Biol. 2010;2(5):243-54.

12. Hao Z, Lou H, Zhu R, Zhu J, Zhang D, Zhao BS, et al. The multiple antibiotic resistance regulator MarR is a copper sensor in Escherichia coli. Nat Chem Biol. 2014;10(1):21-8.

13. Roy A, Ranjan A. HosA, a MarR family transcriptional regulator, represses nonoxidative Hydroxyarylic acid decarboxylase operon and is modulated by 4-Hydroxybenzoic acid. Biochemistry. 2016;55(7):1120-34.

14. Fuangthong M, Helmann JD. The OhrR repressor senses organic hydroperoxides by reversible formation of a cysteine-sulfenic acid derivative. Proc Natl Acad Sci U S A. 2002;99(10):6690-5.

15. Fuangthong $M$, Atichartpongkul S, Mongkolsuk S, Helmann JD. OhrR is a repressor of ohrA, a key organic hydroperoxide resistance determinant in Bacillus subtilis. J Bacteriol. 2001;183(14):4134-41.

16. Antelmann H, Helmann JD. Thiol-based redox switches and gene regulation. Antioxid Redox Signal. 2011;14(6):1049-63.

17. Netto LES, Oliveira MA, Tairum CA, da Silva Neto JF. Conferring specificity in redox pathways by enzymatic thiol/disulfide exchange reactions. Free Radic Res. 2016;50(2):206-45.

18. Atichartpongkul S, Vattanaviboon P, Wisitkamol R, Jaroensuk J, Mongkolsuk S, Fuangthong M. Regulation of organic hydroperoxide stress response by two OhrR homologs in Pseudomonas aeruginosa. PLoS One. 2016;11(8):e0161982. 
19. Lan L, Murray TS, Kazmierczak BI, He C. Pseudomonas aeruginosa OspR is an oxidative stress sensing regulator that affects pigment production, antibiotic resistance and dissemination during infection. Mol Microbiol. 2010;75(1):76-91.

20. Luong TT, Dunman PM, Murphy E, Projan SJ, Lee CY. Transcription profiling of the mgra regulon in Staphylococcus aureus. J Bacteriol. 2006;188(5):1899-910.

21. Crosby HA, Schlievert PM, Merriman JA, King JM, Salgado-Pabón W, Horswill AR. The Staphylococcus aureus global regulator MgrA modulates clumping and virulence by controlling surface protein expression. PLoS Pathog. 2016;12(5):e1005604.

22. Chen PR, Nishida S, Poor CB, Cheng A, Bae T, Kuechenmeister L, et al. A new oxidative sensing and regulation pathway mediated by the MgrA homologue SarZ in Staphylococcus aureus. Mol Microbiol. 2009;71(1):198-211.

23. Liu X, Sun X, Wu Y, Xie C, Zhang W, Wang D, et al. Oxidation-sensing regulator AbfR regulates oxidative stress responses, bacterial aggregation, and biofilm formation in Staphylococcus epidermidis. J Biol Chem. 2013;288(6):3739-52.

24. Teixeira FL, Silva DN, Pauer H, Ferreira LQ, de Oliveira Ferreira E, Domingues RM, et al. The role of BmoR, a MarR family regulator, in the survival of Bacteroides fragilis during oxidative stress. Int J Med Microbiol. 2013;303(8):443-8.

25. Brugarolas P, Movahedzadeh F, Wang Y, Zhang N, Bartek IL, Gao YN, et al. The oxidation-sensing regulator (MosR) is a new redoxdependent transcription factor in mycobacterium tuberculosis. J Biol Chem. 2012;287(45):37703-12.

26. Lebreton F, van Schaik W, Sanguinetti M, Posteraro B, Torelli R, Le Bras F, et al. AsrR is an oxidative stress sensing regulator modulating enterococcus faecium opportunistic traits, antimicrobial resistance, and pathogenicity. PLoS Pathog. 2012;8(8):e1002834.

27. Miki T, Iguchi M, Akiba K, Hosono M, Sobue T, Danbara H, et al. Chromobacterium pathogenicity island 1 type III secretion system is a major virulence determinant for Chromobacterium violaceum-induced cell death in hepatocytes. Mol Microbiol. 2010;77(4):855-72.

28. Miki T, Akiba K, Iguchi M, Danbara H, Okada N. The Chromobacterium violaceum type III effector CopE, a guanine nucleotide exchange factor for Rac1 and Cdc42, is involved in bacterial invasion of epithelial cells and pathogenesis. Mol Microbiol. 2011;80(5):1 186-203.

29. Santos RERS, Batista BB, da Silva Neto JF. Ferric uptake regulator Fur coordinates Siderophore production and defense against Iron toxicity and oxidative stress and contributes to virulence in Chromobacterium violaceum. Appl Environ Microbiol. 2020;86(21):e01620.

30. Previato-Mello M, de Abreu Meireles D, Netto LES, da Silva Neto JF. Global transcriptional response to organic hydroperoxide and the role of OhrR in the control of virulence traits in Chromobacterium violaceum. Infect Immun. 2017:85(8):e00017.

31. Barroso KCM, Previato-Mello M, Batista BB, Batista JH, da Silva Neto JF. EmrR-dependent Upregulation of the efflux pump EmrCAB contributes to antibiotic resistance in Chromobacterium violaceum. Front Microbiol. 2018;9:2756.

32. da Silva Neto JF, Negretto CC, Netto LES. Analysis of the organic hydroperoxide response of Chromobacterium violaceum reveals that OhrR is a cys-based redox sensor regulated by thioredoxin. PLoS One. 2012;7(10):e47090.

33. Batista BB, De Souza Santos RER, Ricci-Azevedo R, Da Silva Neto JF. Production and uptake of distinct endogenous catecholate-type siderophores are required for iron acquisition and virulence in Chromobacterium violaceum. Infect Immun. 2019;87(12):e00577-19.
34. Peralta DR, Adler C, Corbalán NS, Paz García EC, Pomares MF, Vincent PA. Enterobactin as part of the oxidative stress response repertoire. PLoS One. 2016;11(6):e0157799.

35. Rabin RS, Stewart V. Dual response regulators (NarL and NarP) interact with dual sensors (NarX and NarQ) to control nitrate- and nitrite-regulated gene expression in Escherichia coli K-12. J Bacteriol. 1993;175(11):3259-68.

36. Hartig E, Schiek U, Vollack KU, Zumft WG. Nitrate and nitrite control of respiratory nitrate reduction in denitrifying pseudomonas stutzeri by a two-component regulatory system homologous to NarXL of Escherichia coli. J Bacteriol. 1999;181(12):3658-65.

37. Abomoelak B, Hoye EA, Chi J, Marcus SA, Laval F, Bannantine JP, et al. mosR, a novel transcriptional regulator of hypoxia and virulence in mycobacterium tuberculosis. J Bacteriol. 2009;191(19):5941-52.

38. Bussmann M, Baumgart M, Bott M. RosR (Cg1324), a hydrogen peroxidesensitive MarR-type transcriptional regulator of Corynebacterium glutamicum. J Biol Chem. 2010;285(38):29305-18.

39. González PJ, Correia C, Moura I, Brondino CD, Moura JJG. Bacterial nitrate reductases: molecular and biological aspects of nitrate reduction. J Inorg Biochem. 2006;100(5-6):1015-23.

40. Bazylinski DA, Palome E, Blakemore NA, Blakemore RP. Denitrification by Chromobacterium violaceum. Appl Environ Microbiol. 1986;52(4):696-9.

41. Bollag JM, Henninger NM. Effects of nitrite toxicity on soil bacteria under aerobic and anaerobic conditions. Soil Biol Biochem. 1978;10:377-81.

42. Bueno E, Sit B, Waldor MK, Cava F. Anaerobic nitrate reduction divergently governs population expansion of the enteropathogen vibrio cholerae. Nat Microbiol. 2018;3(12):1346-53.

43. Kaihami GH, Breda LCD, Almeida JRF, Pereira TO, Nicastro GG, Boechat $\mathrm{AL}$, et al. The atypical response regulator AtvR is a new player in Pseudomonas aeruginosa. Infect Immun. 2017;85(8):e00207-17.

44. Jiang $Q$, Jin Z, Sun B. MgrA negatively regulates biofilm formation and detachment by repressing the expression of psm operons in Staphylococcus aureus. Appl Environ Microbiol. 2018;84(16):e01008.

45. Tamber S, Cheung AL. SarZ promotes the expression of virulence factors and represses biofilm formation by modulating SarA and agr in Staphylococcus aureus. Infect Immun. 2009;77(1):419-28.

46. Berne C, Ducret A, Hardy GG, Brun YV. Adhesins involved in attachment to abiotic surfaces by negative bacteria. Microbiol Spectr. 2015;3(4):MB-0018-2015.

47. Hanahan D. Studies on transformation of Escherichia coli with plasmids. J Mol Biol. 1983;166(4):557-80.

48. Simon $\mathrm{R}$, Priefer $\mathrm{U}$, Puhler A. A broad host range mobilization system for in vivo genetic engineering: transposon mutagenesis in gram-negative bacteria. Bio/Technology. 1983;1:784-91.

49. Newman JR, Fuqua C. Broad-host-range expression vectors that carry the L-arabinose-inducible Escherichia coli araBAD promoter and the araC regulator. Gene. 1999;227(2):197-203.

50. Fisher RA. Statistical Methods for Research Workers. 4th ed. London; 1932.

51. Cortez DA, Tonon AP, Colepicolo P, Vêncio RZN. Combining P values to improve classification of differential gene expression in the HTself software. Genet Mol Res. 2011;10(4):3586-95.

\section{Publisher's Note}

Springer Nature remains neutral with regard to jurisdictional claims in published maps and institutional affiliations. 